\title{
Variabilidad genética de dos subpoblaciones de Cuyes (Cavia porcellus) nativos del sur del Ecuador
}

\author{
Genetic variability of two sub-populations of native Guinea Pig \\ (Cavia porcellus) from southern Ecuador
}

\author{
Cornelio Rosales-Jaramillo ${ }^{\text {* }}$ iD, Pedro Nieto-Escandón ${ }^{1}$ iD, Rafael Román-Bravo² ${ }^{\text {iD }}$ y José Aranguren-Méndez ${ }^{2}$ iD \\ ${ }^{1}$ Facultad de Ciencias Agropecuarias, Universidad de Cuenca, Ecuador. ${ }^{2}$ Laboratorio de Genética Molecular, Facultad de Ciencias \\ Veterinarias, Universidad del Zulia, Venezuela. Correo electrónico: cornelio.rosales@ucuenca.edu.ec
}

\begin{abstract}
RESUMEN
La creciente necesidad actual de conservar los recursos zoogenéticos locales como fuentes de variabilidad genética (VG), plantea la necesidad de realizar investigaciones que ayuden a conocer su estado actual; por ello, se estudió la VG de dos subpoblaciones nativas de cuyes (Cavia porcellus) denominadas: Azuay y Cañar, geográficamente ubicadas al sur de Ecuador. Para su caracterización se utilizaron seis marcadores microsatélites de ADN, muestreándose 50 animales de cada subpoblación y un tercer grupo de 50 animales de origen peruano como grupo externo para comparación. De los seis marcadores usados, cinco pudieron ser amplificados; se encontró un número de alelos considerable $(9,8)$ en la población general y un valor de $(7,02)$ igual en ambas poblaciones con un alto grado de polimorfismo ( $\mathrm{PIC}=0,7035)$; dos loci estudiados, el MS I en ambas subpoblaciones y MS III en Azuay no se encontraron en equilibrio HW. La VG variabilidad fue alta en ambas subpoblaciones $\left(\mathrm{H}_{\circ} 0,694\right)$, así como un cierto grado de diferenciación genética $\left(\mathrm{G}_{\mathrm{st}}=0,066\right)$, existiendo dos alelos privados con frecuencias superiores al $10 \%$ (MS IV, Azuay 301 pb y 297 pb en Cañar), dando indicio de constituirse como marcadores de raza. La distancia genética entre subpoblaciones Azuay y Cañar es media $(0,17)$, no obstante, resultó ser superior a las encontradas entre cada una de éstas y la población Perú. La realidad genética encontrada sugiere la necesidad de intervenir sobre estas poblaciones con la finalidad de conservar el material genético nativo incorporando un manejo sostenible del recurso zoogenético.
\end{abstract}

Palabras clave: Cuy; microsatélite; variabilidad genética; subpoblación nativa; Ecuador

\begin{abstract}
The current growing need to conserve local animal genetic resources as sources of genetic variability (GV), raises the need to carry out research that helps to know their current state; for this reason, the GV of two native subpopulations of guinea pigs (Cavia porcellus) called: Azuay and Cañar, geographically located in the south of Ecuador, was studied. For its characterization, six-microsatellite DNA markers were used, sampling 50 animals from each subpopulation and a third group of 50 animals of Peruvian origin as an external group for comparison. Of the six markers used, five could be amplified; a considerable number of alleles (9.8) was found in the general population and a value of (7.02) the same in both populations with a high degree of polymorphism (PIC $=0.7035)$; two loci studied, MS I in both subpopulations and MS III in Azuay, were not found in HW equilibrium. GV was high in both subpopulations $\left(\mathrm{H}_{\circ} 0.694\right)$, as well as a certain degree of genetic differentiation $\left(\mathrm{G}_{\mathrm{st}}=0.066\right)$, with two private alleles with frequencies above $10 \%$ (MS IV, Azuay 301 bp and 297 bp in Cañar), giving an indication of becoming markers of race. The genetic distance between Azuay and Cañar subpopulations is medium (0.17), however, it turned out to be higher than those found between each of these and the Peruvian population. The genetic reality found suggests the need to intervene on these populations in order to conserve the native genetic material incorporating a sustainable management of the zoogenetic resource.
\end{abstract}

Key words: Guinea pig; microsatellites; genetic variability; native subpopulation; Ecuador 
Variabilidad genética de dos subpoblaciones de Cuyes / Rosales-Jaramillo y col.

\section{INTRODUCCIÓN}

El cuy (C) o cobayo (Cavia porcellus) es un roedor histricomorfo de la familia Caviidae, originario de los Andes, cuya domesticación se calcula ocurrió entre los 6.000 y 2.000 años antes de cristo (AC) $[8,15,16]$. Investigaciones de su secuencia genómica indican que el $\mathrm{C}$ actual podría derivarse de diferentes troncos, a partir de especies diferentes y domesticadas en regiones geográficas distintas. Es así, que se identifican núcleos de posible domesticación, en Colombia derivado del C. anolaimae [16], en el sur de Perú y norte de Chile basado en el C. tschudii [29] y en la región central Ecuador de la especie C. patzelti [9].

Los recursos zoogenéticos son la raíz de la ganadería moderna y se definen como la diversidad de animales que proporcionan múltiples productos, fuerza de trabajo y otros beneficios que contribuyen a solventar las necesidades humanas [20]. La gran diversidad genética (DG) generada de manera natural, se vio afectada a principios del siglo XIX con el incremento de los procesos de intensificación de la producción animal, fundamentada en la movilización de reproductores y el inicio de los llamados programas de "mejoramiento genético", lo que conllevó a la selección y cría de poblaciones de animales con características productivas especiales, como la alta producción a los que se definieron como "razas puras", concepto actualmente en discusión ya que son variedades domésticas generadas por la manipulación humana y que son altamente endogámicas constituyendo islas genéticas que evitan que los genes fluyan de un acervo genético a otro dentro de la misma especie, lo que ocasiona una gran pérdida de la DG [28, 33].

Esta pérdida de la DG en las especies domésticas, implica la disminución de su capacidad de adaptación a cambios, ya sean naturales, como el cambio climático o antrópicos, ocasionados por los procesos de domesticación y selección reproductiva, aplicados para alcanzar mayores índices de producción [31]. La Organización de las Naciones Unidas para la Alimentación y la Agricultura (FAO) advierte que, muchas razas de animales domésticos se encuentran amenazadas por un uso no sostenible del patrimonio genético, existiendo un $17 \%$ de éstas en el mundo, que están en peligro de extinción y de ellas un $58 \%$ están en situación de riesgo, por desconocer su realidad actual, destacándose que entre los años 2000 y 2014 , cerca de 100 razas de ganado se extinguieron [12].

Uno de los principales factores que amenazan la diversidad de los animales domésticos nativos, es la importación o sustitución de las razas locales por razas mejoradas, consideradas más productivas, situación que conduce a menudo al cruce absorbente de estas razas, hasta la sustitución total de los animales nativos [1]. Resulta entonces fundamental el cuidado del material genético local por sus características de resiliencia debiendo conservarse la DG en base a cruzamientos racionales, lo que implica un manejo sostenible de la especie sin descuidar el incremento de la producción y productividad dentro del manejo zootécnico [19, 23, 32].

El C, como especie doméstica por su importancia cultural y alimenticia en el mundo andino, al proporcionar identidad y seguridad alimentaria además de su proyección mundial actual, no obstante, se ha visto afectado desde los años 50, por un proceso de mejoramiento genético continuo, generándose líneas de mayor productividad las que han invadido en los países andinos por proporcionar rendimientos económicos más altos [25]. Como podía preverse de manera simultánea, los animales nativos están siendo desvalorizados aceleradamente y reemplazados por animales genéticamente mejorados, sin considerar que dichos animales son componentes de la cultura, sistemas económicos tradicionales, paisajes y ecosistemas [10, 11].

El riesgo inminente de la perdida de la VG, a futuro podría poner en riesgo a la especie al desaparecer las poblaciones nativas que son reservorios naturales de genes que les dotan de una amplia capacidad de resiliencia, adaptabilidad, resistencia y rusticidad entre otros. Los esfuerzos para conformar núcleos de animales criollos o nativos y posterior evaluación deben ser prioritarios, principalmente en los países en desarrollo, donde hay incertidumbre acerca de las condiciones futuras de producción y de mercado, además que representan un recurso alternativo para mantener la producción animal bajo cualquier cambio drástico de tipo ambiental o económico [17].

Estudios previos, comparando poblaciones criollas versus (vs), poblaciones mejoradas, indican que los porcentajes de DG en Cavia sugieren una notable pérdida de variabilidad en las poblaciones criollas [27]. Los valores bajos de variabilidad en la población mejorada, se explican debido al efecto ejercido por la selección continua de genotipos para fines comerciales lo que ha conducido a una homogenización genética. Mientras que, en el caso de las poblaciones locales o criollas, la erosión genética se debió principalmente a que correspondían a núcleos cerrados, pertenecientes a los grupos genéticos conservados, sin la posibilidad de llevar a cabo procesos de diversificación, principalmente por la dificultad de encontrar animales nativos en la zona, para utilizarlos como reproductores, conllevando a apareamientos entre individuos relacionados. Por lo tanto, la pérdida de la DG natural de $C$. porcellus en la región es evidente debido a la absorción del material genético autóctono por el C peruano [27].

Uno de los principales problemas que afectan a estas poblaciones nativas minoritarias, son los apareamientos entre individuos emparentados, que se traducen genéticamente en un incremento de la consanguinidad (homocigosis) con la consiguiente depresión endogámica, es decir una reducción de los valores medios fenotípicos de los caracteres productivos y reproductivos, y, por último, como consecuencia de esos problemas reproductivos, la inevitable disminución y/o extinción de la población [2].

De allí que el principal objetivo de este artículo fue estudiar la VG en dos poblaciones nativas de $\mathrm{C}$ del sur del Ecuador, a través del análisis de un conjunto de microsatélites, y evaluar el grado de diferenciación genética existente entre estas dos variedades y otra raza mejorada del Perú, todo ello enmarcado como punto de inicio para la futura puesta en marcha de un programa de conservación genética de esta especie.

\section{MATERIALES Y MÉTODOS}

\section{Muestras de las subpoblaciones y extracción de ácido desoxirribonucleico (ADN)}

Para la investigación se obtuvieron muestras aleatorias de 100 $C$ nativos (C. porcellus) de ambos sexos y no emparentados, 25 machos y 25 hembras pertenecientes a la subpoblación Azuay e igual cantidad de la subpoblación Cañar, ubicadas geográficamente en el altiplano sur del Ecuador (FIG. 1). Además, se muestrearon $50 \mathrm{C}$ mejorados de origen peruano como grupo externo de comparación (out-group). EI ADN se obtuvo a partir de muestras de sangre, mediante punción de la vena cefálica 


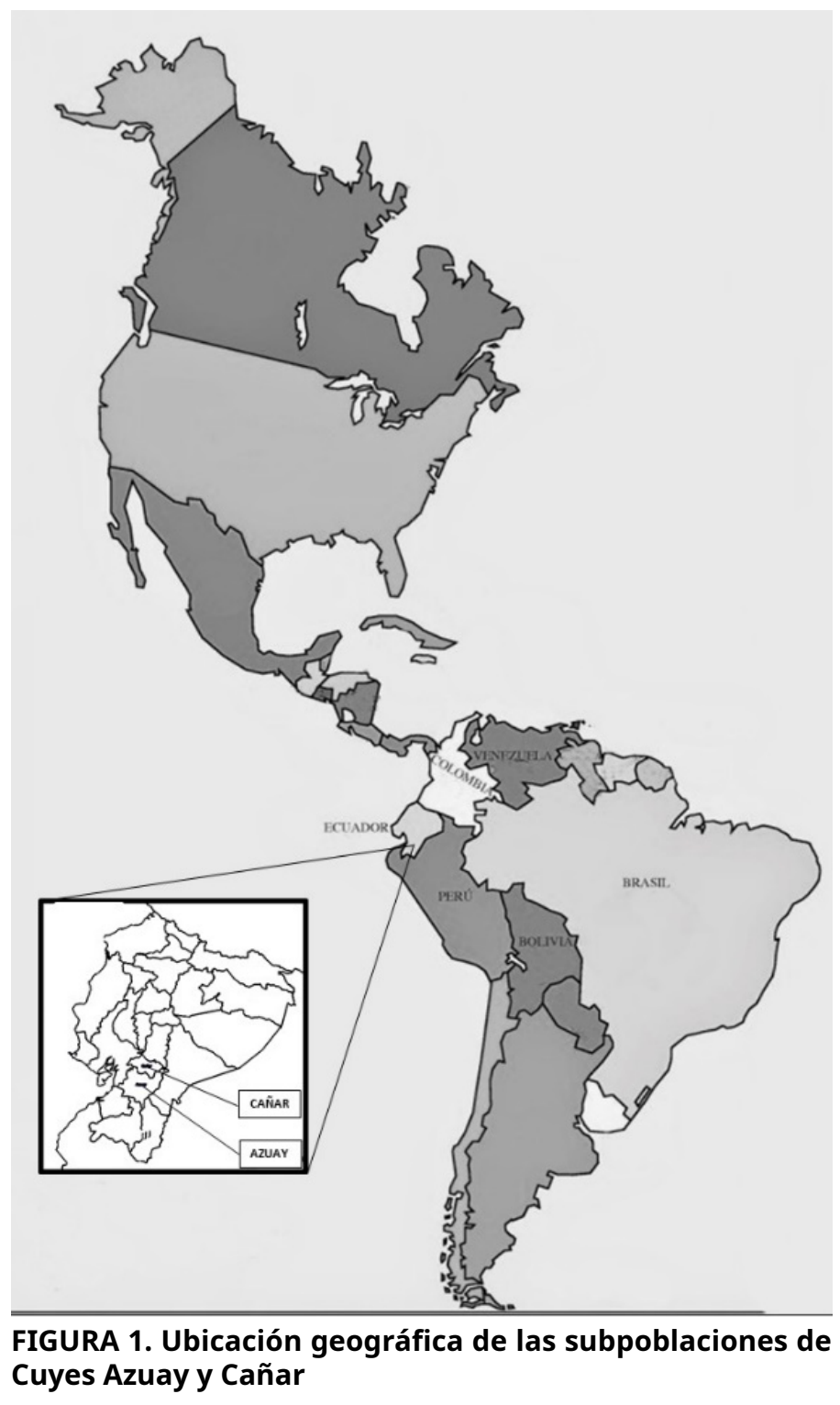

de 2 mililitros $(\mathrm{mL})$ y colocada en tubos con anticoagulante ácido etilendiaminotetraacético (EDTA), y procesada mediante el método estándar que incluye lisado celular y la extracción con mezcla de fenol:cloroformo:isoamiloalcohol, en una proporción 25:24:1 [4].

\section{Marcadores microsatélites}

Se utilizaron un total de 6 marcadores microsatélites, reportados previamente para $C$. aperea e identificados como MS I, MS II, MS III, MS IV, MS V y MS VI [3]. Las secuencias de los cebadores y su número de acceso al Genbank se aprecia en la TABLA I.

\section{Análisis de la reacción en cadena de la polimerasa (PCR)}

Las PCR fueron llevadas a cabo en una reacción final con 25 microlitros $(\mu \mathrm{L})$, que contenía 30 nanogramos (ng) de ADN (genómico), 200 micro mol $(\mu \mathrm{M})$ de dNTP (desoxinucleótidos), $0,5 \mu \mathrm{L}$ de la enzima polimerasa, 1,5 mili $\mathrm{Mol}(\mathrm{mM})$ de $\mathrm{MgCL}_{2}$ y
TABLA I

Microsatélites y secuencias de los seis marcadores utilizados

\begin{tabular}{clc}
\hline Locus & \multicolumn{1}{c}{ Secuencias $\left(\mathbf{5}^{\prime}\right.$ - $\mathbf{3}$ ') } & $\begin{array}{c}\text { GENBANK } \\
\text { Access }\end{array}$ \\
\hline \multirow{2}{*}{ MS I } & $\begin{array}{l}\text { F: ATT GGC TTC ATT GCT ATG GAC } \\
\text { R: GGC CTG CTC CTG TTC TC }\end{array}$ & $\underline{\text { AJ496558 }}$ \\
MS II & $\begin{array}{l}\text { F: AGA AGC CAG CTC TGG ATT C } \\
\text { R: GCA TCC ACA GAA TGT GGA TC }\end{array}$ & $\underline{\text { AJ496559 }}$ \\
& F:GGC CAT TAT GCC CCC CAA C & AJ496560 \\
MS III & R:AGC TGC TCC TTG TGC TGT AG & $\underline{ }$ \\
MS IV & $\begin{array}{l}\text { F:CTT CCA CAG CGA TCA CAA TC } \\
\text { R:TTG ACG AAC GCC AGT GTG C }\end{array}$ & $\underline{\text { AJ496561 }}$ \\
MS V & $\begin{array}{l}\text { F:TTC CTT TAC TGG TTT GGA GG } \\
\text { R:ATG GTA GGC ACT TCC ACT G }\end{array}$ & $\underline{\text { AJ496562 }}$ \\
MS VI & F: GGT AAG CTT TTG GGA TTG AGG & AJ496563 \\
\hline
\end{tabular}

$0,20 \mu \mathrm{L}$ de cada uno de los cebadores. Para la amplificación, se llevó a cabo en un termociclador Eppendorf Mastercycler® nexus GSX1 (Hamburgo, Alemania), mediante un programa térmico que consistió en una fase de desnaturalización inicial a $94^{\circ} \mathrm{C}$ durante 5 minutos ( $\mathrm{min}$ ), seguida por una fase de 40 ciclos, conformados por desnaturalización a $94^{\circ} \mathrm{C}$ por 30 segundos (seg), hibridación a $52,3^{\circ} \mathrm{C}$ durante $25 \mathrm{seg}$, extensión cíclica a $72^{\circ} \mathrm{C}$ por $30 \mathrm{seg}$ y una fase de extensión final a $72^{\circ} \mathrm{C}$ durante $5 \mathrm{~min}$.

Los productos amplificados, luego fueron analizados mediante electroforesis capilar con un analizador genético automático de ADN Applied Biosystems ABI Prism® 310 (Estados Unidos de Norteamérica, California), e interpretados posteriormente mediante el software GeneMaper ID v3.2. [13].

\section{ANÁLISIS ESTADÍSTICO}

Se estudiaron los estadísticos convencionales de VG, tales como, el número total de alelos y su rango, contenido de información polimórfica (PIC) y probabilidad de no exclusión por padre (PNE$1 \mathrm{P})$, para cada uno de los loci y el total de la población estudiada, los cuales fueron calculados utilizando el software Cervus 3.0.7 [18]. La heterocigosidad esperada $\left(\mathrm{H}_{\mathrm{e}}\right)$ y observada $\left(\mathrm{H}_{\mathrm{o}}\right)$, para cada uno de los loci polimórficos fueron obtenidas usando el programa Byosis-2 [30]. El test de frecuencias genotípicas para el equilibrio Hardy-Weinberg (EHW) fue calculado utilizando el método del test exacto, mediante el programa GENEPOP 3.1 [24].

Para el estudio de las relaciones filogenéticas entre las subpoblaciones y la posterior construcción del dendrograma, se utilizó la distancia DA de Nei [21] y el algoritmo NJ - neighborjoining-method [26], usando para ello el programa DISPAN [22].

\section{RESULTADOS Y DISCUSION}

Todos los marcadores amplificaron exitosamente en los $\mathrm{C}$ ecuatorianos, excepto el locus MS II que no logró amplificar. La población de animales nativos mostró un número de alelos considerable, con un promedio de $9,8 \pm 3,6$, oscilando dichos valores entre 7 y 16 en los loci MS III y MS IV, respectivamente, diferenciándose marcadamente este último de los otros cuatro 
(TABLA II), no existiendo diferencias entre el número de alelos promedio en las dos subpoblaciones Azuay y Cañar, con valores en ambos casos, de 7,02 $\pm 1,0$. Siendo el número exacto de alelos por loci de 7 vs 6, para el marcador MS I; 4 vs 7 para el MS III; 10 vs 11 para el MS IV; 7 vs 7 para el MS V y 8 vs 5 para el MS VI, respectivamente.

El número de alelos encontrado en los diferentes locus, se ubican en un rango de 7 a 9 excepto en el MS IV que es mucho mayor; el valor promedio 9,8 obtenido, resultó menor a lo reportado para una muestra de animales nativos del altiplano de Ecuador, investigación que tomó en cuenta un mayor número de microsatélites incluidos dos de los estudiados [5], aunque fue superior a lo encontrado en $C$ nativos colombianos [7] y muy superior a lo encontrado en las poblaciones de $\mathrm{C}$ estudiadas en Camerún [6] y Costa de Marfil [14], posiblemente estos dos últimos estuvieron bajo efecto de posibles apareamientos consanguineos. La no amplificacion del marcador MSII pudo deberse a que se trabajó con una especie diferente para la que originalmente fue diseñado (C. aperea), aspecto encontrado también por Burgos y col. [7], confirmándose que dicho marcador no debe ser usado para estudios en C. porcellus.

La heterocigosis total $\left(\mathrm{H}_{\mathrm{t}}\right)$ de la subpoblación nativa como indicador de la diversidad genética, obtuvo una media de $0,742 \pm 0,069$, encontrándose valores para los loci que varían entre 0,666 para el marcador MS V y 0,841 (MS IV). La media de la heterocigosis esperada $\left(\mathrm{H}_{\mathrm{e}}\right)$ en la muestra total fue de 0,694 $\pm 0,078$ oscilando los valores entre 0,608 (MS III) y 0,804 (MS IV). En cuanto al coeficiente promedio de diferenciación genética (Gst) se observó que alcanza el valor de 0,066 \pm 0,039 para el total de loci estudiados; en los loci individuales los valores variaron entre 0,031 para MS I y 0,117 para MS III, siendo precisamente estos los que muestran diferencias significativas $(P<0,01)$ en el test de $\mathrm{H}-\mathrm{W}$. El PIC en los diferentes loci fluctúa entre 0,627 para MS III y 0,822 para MS IV; y la probabilidad de no exclusión combinada para padre (PNE-1P) fue de 0,107.
Estos valores promedios de $\mathrm{H}_{\mathrm{e}}$ para el total de la población estudiada fue similar a los valores reportados para $C$ nativos [5, 7]. EI PIC, mostró que todos los loci son considerados polimórficos y altamente informativos $(0,7035 \pm 0,107)$, en virtud que sobrepasaron el rango teórico de 0,5 , lo que indica una alta diversidad génica en estas subpoblaciones concordante con otros trabajos que utilizaron de manera similar marcadores del tipo microsatélites, para estudios poblacionales [7].

La diferenciación genética promedio (Gst) de las subpoblaciones alcanzó el valor de 6,6 \%, producto de la contribución de todos los loci en especial del MS III y MS VI, esto indica que entre las subpoblaciones nativas estudiadas existe un nivel de diferenciación genética, pero sin llegar a ser alto, no obstante, este valor resultó ser mayor que el encontrado en un trabajo previo en $\mathrm{C}$ ecuatorianos [5].

El HWE fue probado para ambas subpoblaciones y los 5 marcadores polimórficos. De los 10 contrastes o combinaciones grupo genético-loci, 3 dieron desviaciones significativas del equilibrio HWE, mostrando 2 de ellas exceso de heterocigosis (marcador MS I en ambos grupos y el MS III déficit en la subpoblación Azuay), los demás marcadores (MS IV, MS V y MS VI) mostraron equilibrio HW en ambos grupos genéticos.

Esta "alta" VG encontrada en las subpoblaciones nativas de Azuay y Cañar, se puede explicar debido a que territorialmente no se encuentran demasiado restringidas, existiendo aún una población de animales distribuida entre criadores tradicionales de edad avanzada que valoran las características de rusticidad así como las organolépticas de la carne de $\mathrm{C}$ nativo, quienes a través de los mercados tradicionales pueden intercambiar animales, lo que favorecería el flujo genético manteniendo un grado alto de VG, este criterio se ve fortalecido al considerar que solamente uno de los loci no se encontró en equilibrio HW en ambas subpoblaciones nativas estudiadas, lo que indica que estas subpoblaciones aún no han sido afectadas dramáticamente por los procesos de absorción genética, por la introducción de líneas mejoradas.

TABLA /I

Número total y rango de alelos observados, media de heterocigosis $\mathrm{H}_{\mathrm{t}}$ y $\mathrm{H}_{\mathrm{e}}$, coeficiente de diferenciación Gst, H-W, PIC y PNE de la población total de Cuyes nativos ecuatorianos

\begin{tabular}{|c|c|c|c|c|c|c|c|c|}
\hline Microsatélite & $N^{\circ} A^{1}$ & Rango $^{2}$ & $\mathbf{H}_{\mathrm{t}^{3}}$ & $\mathrm{He}^{4}$ & $\mathbf{G S T}^{5}$ & $H-W^{6}$ & PIC $^{7}$ & PNE-1P8 \\
\hline MS I & 9 & $209-293$ & 0,761 & 0,737 & 0,031 & $\star \star \star$ & 0,721 & 0,642 \\
\hline MS III & 7 & $133-151$ & 0,688 & 0,608 & 0,117 & $\star \star$ & 0,627 & 0,738 \\
\hline MS IV & 16 & $243-315$ & 0,841 & 0,804 & 0,044 & NS & 0,822 & 0,481 \\
\hline MS V & 9 & $143-193$ & 0,666 & 0,639 & 0,040 & NS & 0,629 & 0,735 \\
\hline MS VI & 8 & $153-171$ & 0,755 & 0,680 & 0,099 & NS & 0,718 & 0,637 \\
\hline Todos & 9,8 & & 0,742 & 0,694 & 0,066 & & 0,7035 & 0,107 \\
\hline DS & $\pm 3,6$ & & $\pm 0,069$ & $\pm 0,078$ & $\pm 0,039$ & & & \\
\hline
\end{tabular}

${ }^{1}$ Número total de alelos observados; ${ }^{2}$ Rango de alelos observados (pb); ${ }^{3}$ Promedio de heterocigosis esperada población total; ${ }^{4}$ Promedio heterocigosis esperada; ${ }^{5}$ Coeficiente de diferenciación; ${ }^{6}$ Equilibrio Hardy-Weinberg; ${ }^{7}$ Contenido de información polimórfica; ${ }^{8}$ Probabilidad de no exclusión (padre) 
En las poblaciones de $\mathrm{C}$ del sur del Ecuador, el número medio de alelos por locus fue similar en ambos grupos genéticos, no obstante, la heterocigosidad promedio observada presentó valores de 0,676 $\pm 0,100$ en Azuay y de 0,746 $\pm 0,071$ en Cañar, entre tanto que la heterocigosidad esperada correspondió a valores de $0,688 \pm 0,051$ y $0,713 \pm 0,026$ en el mismo orden (TABLA III).

\section{TABLA III}

Tamaño de muestra, promedio y error estándar de número de alelos y heterocigosidad sobre seis microsatélites por subpoblación de Cuyes nativos ecuatorianos

\begin{tabular}{ccccc}
\hline & \multirow{2}{*}{$\begin{array}{c}\text { Tamaño } \\
\text { Población }\end{array}$} & $\begin{array}{c}\text { Promedio de } \\
\text { muestras } \\
\text { por locus }\end{array}$ & $\begin{array}{c}\text { alelos } \\
\text { por locus }\end{array}$ & \multicolumn{2}{c}{$\begin{array}{c}\text { Promedio de } \\
\text { heterocigosidad }\end{array}$} \\
\cline { 4 - 6 } Azuay & 50 & $7,2 \pm 1,0$ & $0,676 \pm 0,100$ & $0,688 \pm 0,051$ \\
Cañar & 48 & $7,2 \pm 1,0$ & $0,746 \pm 0,071$ & $0,713 \pm 0,026$ \\
\hline
\end{tabular}

El análisis de identificación de alelos mostró poca diferencia entre las dos subpoblaciones de $\mathrm{C}$ del sur de Ecuador estudiadas (FIG. 2). A pesar de que se presentaron algunos alelos privados dentro de cada subpoblación, éstos siempre fueron en frecuencias bajas, indicando, posiblemente que este hecho sea producto único y exclusivamente del bajo número de individuos analizados.
El número de alelos únicos (privados) encontrados en la subpoblación Azuay fue 3 en el marcador MS I (213, 217 y 219 pb), 5 en MS IV $(243,255,259,287$ y 301 pb), 2 en MS V (157, 159 pb) y 3 en MS VI (171, 153 y 159 pb), por el contrario, en Cañar se determinó 2 en M SI (241, 243 pb), 3 en MS III (133,149 y 151 pb), 6 en MS IV $(265,269,283,297,311$ y 315 pb) y 2 en MS V (143 y 193 pb); solo dos alelos superaron el 10 \% (MS IV; alelo de 301 pb con una frecuencia de $12 \%$ en la subpoblación Azuay y el alelo de 297 pb del mismo microsatélite, con una frecuencia del $24 \%$ en la subpoblación Cañar).

El porcentaje de diferencia genética encontrado, está influenciado por la existencia de alelos privados, algunos con una frecuencia superior al $10 \%$ (MS IV; alelo de 301 pb en Azuay y alelo 297 pb en Cañar), siendo relevante el encontrado en la población Cañar con una frecuencia del $24 \%$, lo que hace suponer que puede constituirse en un marcador de la raza, aún por confirmar en futuros estudios de paternidades. El valor promedio de probabilidad de no exclusión para paternidad (PNE-1P) alcanza el 10,7 \% siempre que se utilice el conjunto de loci por lo que hay una alta probabilidad de reconocimiento paternal.

La TABLA IV muestra los valores de distancia genética $\left(D_{A}\right.$ de Nei) entre las dos subpoblaciones de $C$ estudiadas y se añadió la variedad mejorada del Perú, esta última utilizada como "outgroup" o población de referencia, con la idea de darle una orientación evolutiva al estudio. La distancia genética determinada entre las dos subpoblaciones de $C$ nativos estudiadas alcanza el valor de 0,1783 , mientras que, las distancias entre las dos subpoblaciones ecuatorianas y la mejorada del Perú, osciló entre 0,1224 y 0,1349, para la subpoblación Cañar y la Azuay, respectivamente.
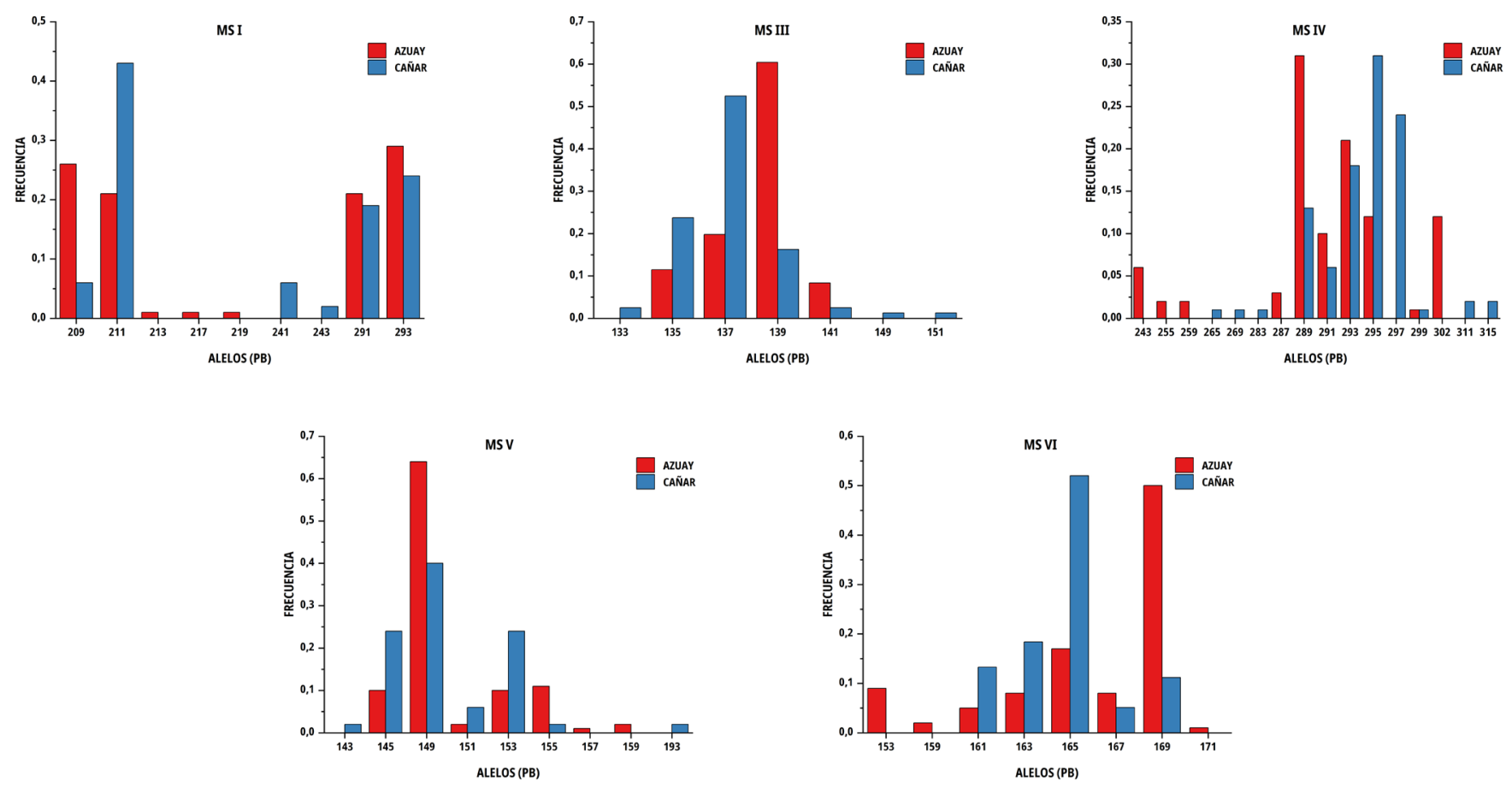

FIGURA 2. Distribución de las frecuencias alélicas para los marcadores MS I, MS III, MS IV, MS V y MS VI en dos subpoblaciones de Cuyes del sur del Ecuador 
TABLA IV

Matriz de distancias genéticas $\left(D_{A}\right)$ entre dos subpoblaciones nativas de Cuyes del sur del Ecuador y un grupo externo mejorado de origen peruano

\begin{tabular}{ccc}
\hline & Azuay & Cañar \\
\hline Cañar & 0,1783 & \\
Perú & 0,1349 & 0,1224 \\
\hline
\end{tabular}

A partir de estas distancias genéticas, se construyó un dendrograma, utilizando para ello el algoritmo NJ (FIG. 3). Las distancias muestran una relación similar entre las dos subpoblaciones nativas y la de origen peruano siendo mayor con Cañar; sin embargo, la distancia genética encontrada entre las dos subpoblaciones nativas fue considerablemente mayor, existiendo una menor relación entre ellas; no obstante, estos valores inferiores a 0,20 dan indicio de una cercanía próxima entre los 3 grupos.

La distancia genética entre las dos subpoblaciones nativas se observa de valores medio $(0,1783)$, demostrando muy probable el origen común de las dos subpoblaciones Azuay y Cañar, con posterior desarrollo de grupos poblacionales diferentes que se desarrollaron en cada zona. Al introducir para el análisis un grupo externo de origen peruano se observa que las distancias genéticas disminuyen entre las dos subpoblaciones frente a éste, lo que pudiera indicar que se presenta un origen común con el $\mathrm{C}$ peruano, encontrándose más cercana la subpoblación Cañar a Perú.

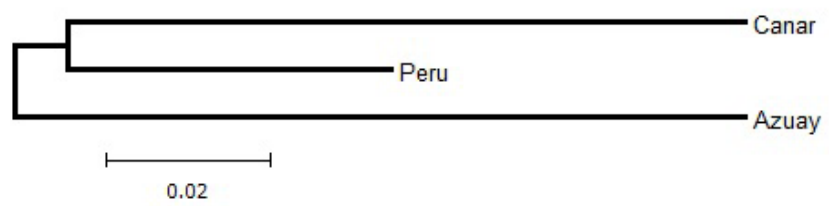

FIGURA 3. Dendrograma con $D_{A}$ y NJ de relaciones genéticas entre dos subpoblaciones nativas de Cuyes ecuatorianos y una subpoblación de origen peruano

\section{CONCLUSIONES}

Del análisis de estas dos subpoblaciones nativas de C: Azuay y Cañar, se observó que mantiene un alto grado de VG con $\mathrm{He}$ de 0,7 y 7,2 alelo por locus, factor que debe ser cuidado y preservado con visión hacia el futuro.

Estas subpoblaciones se encuentran relacionadas, pero conservan un porcentaje medio de diferenciación genética propia para cada una de ellas, con valores cercanos de distancia genéticas de 0,17.

Se reconfirma que el uso de secuencias de ADN, tales como los microsatélites resultan ser herramientas valiosas para el estudio de la VG de poblaciones animales, con beneficios potenciales para la conservación de los recursos zoogenéticos locales.

\section{AGRADECIMIENTO}

A la Dirección de Investigación de la Universidad de Cuenca (DIUC), Cuenca-Ecuador quien financió el proyecto "Caracterización molecular y fenotípica de ecotipos de cobayos criollos a partir de la recuperación y formación de núcleos de crianza ex situ como base para la formación de una línea genética local". Así mismo, agradecemos las sugerencias de los árbitros.

\section{CONFLICTOS DE INTERESES}

Los autores declaran que no tienen conflictos de intereses en la investigación.

\section{REFERENCIAS BIBLIOGRAFICAS}

[1] ANZOLA, H. Conservación y Utilización de las Razas Bovinas Criollas y Colombianas para el Desarrollo Rural Sostenible. Arch. Zoot. 54(206-207): 141-144. 2005.

[2] ARANGUREN, J.; JORDANA, J.: AVELLANET, R.; TORRENS, $M$. Estudio de la Variabilidad Genética en la Raza Bovina Mallorquina para Propósitos de Conservación. Rev. Cientif. FCV-LUZ XII(5): 358-366. 2002.

[3] ASHER, M.; LIPPMANN, T.; EPPLEN, T.; KRAUS, C.; TRILLMICH, F.; SACHSER, N. Large males dominate: ecology, social organization, and mating system of wild cavies, the ancestors of the guinea pig. Behav. Ecol. Sociobiol. 62(9): 1509-1521. 2008.

[4] AUSUBEL, F.; BRENT, R.; KINGSTON, R.; MOORE, D.; SEIDMAN, J.; SMITH, J.; STRUHL, K. Current Protocols in Molecular Biology. Struhl, K. (Ed.). Pp 198-200. 2003.

[5] AVILÉS, D. Caracterización genética del cuy doméstico en América de Sur mediante marcadores moleculares. Universidad de Córdova, Córdova-España. Tesis de Doctorado. 115 pp. 2016

[6] AYAGIRWE, B.; MEUTCHIEYE, F.; DJIKENG, A.; SKILTON, R.; OSAMA, S.; MANJELI, Y. Genetic Diversity and Structure of Domestic Cavy (Cavia porcellus) Populations from Smallholder Farms in Southern Cameroon. Anim. Prod. 19(1): 1-12. 2017.

[7] BURGOS, W.; CERÓN, M.; SOLARTE, C. Genetic diversity and population structure of the Guinea pig (Cavia porcellus, Rodentia, Caviidae) in Colombia. Genet. Mol. Biol. 34(4): 711-718. 2011.

[8] DIGARD, J. Un Aspect méconnu de l'histoire de l'Amérique: la domestication des animaux. L'Homme. 32(122-124): 253-270. 1992.

[9] DUNNUM, J; SALAZAR, B. Molecular systematics, taxonomy and biogeography of the genus Cavia (Rodentia: Caviidae). J. Zool. Syst. Evol. Res. 48(4): 376-388. 2010.

[10] ESTRELLA, J; MANOSALVAS, R.; MARIACA, J.; RIBADENEIRA, M. Biodiversidad y Recursos Genéticos: Una guía para su uso y acceso en el Ecuador. Producciones Digitales Abya-Yala (Ed.) Quito. 116 pp. 2005.

[11] GeneMapper ID (v3.2.) [software]. (2006). Applied Biosystems. 
[12] KOUAKOU, P.; SKILTON, R.; APOLLINAIRE, D.; AGATHE, F.; BEATRICE, G.; CLÉMENT, A. Genetic diversity and population structure of cavy (Cavia porcellus $L$ ) in three agro ecological zones of Côte d'Ivoire. Inter. J. Agron. Agric. Res. 6(3): 27-35. 2015.

[13] LAVALLEÉ, D. La Domestication Animales En Amèrique Du Sud - Le point des connaissances. Bull. Inst. fr. Ėtudes Andines. 19(1): 25-44. 1990.

[14] LORD, E.; COLLINS, C.; DE FRANCE, S.; LEFEBVRE, M.; PIGIÉRE, F.; EECKHOUT, P.; ERAUW, C.; FITZPATRICK, S.; HEALY, P.; MARTÍNEZ, M.; GARCÍA, J.; RAMOS, E.; DELGADO, M.; SÁNCHEZ, A.; PEÑA, G.; TOYNE, J.; DAHLSTEDT, A.; MOORE, K.; LAGUER, C.; ZORI, C.; MATISOO, E. Ancient DNA of Guinea Pigs (Cavia spp.) Indicates a Probable New Center of Domestication and Pathways of Global Distribution. Scientif. Rep. 10(8901): 1-9. 2020.

[15] MANTILLA, J. Mejoramiento Genético y Conservación de Cuyes Nativos en el Perú. Sistema de Revisiones en Investigación Veterinaria de San Marcos. Cajamarca, 08/12-14 Perú. Pp 1-5. 2009.

[16] MARSHALL, T.; SLATE, J.; KRUUK, L.; PEMBERTON, J.M. Statistical confidence for likelihood-based paternity inference in natural populations (CERVUS 3.0.7). Mol. Ecol.7(5): 639-655.1998.

[17] MEZA, E.; RAYMONDI, J.; CISNEROS, S. Evaluación Genética de un plantel de Cuyes Reproductores de Genotipo Perú. Rev. Invest. Vet. Perú. 28(2): 293-298. 2017.

[18] MUJICA, F. Diversidad y Conservación de los Recursos Zoogenéticos del País. Agro Sur. 37(3): 134-175. 2009.

[19] NEI, M; TAJIMA, F.; TATENTO, T. Accuracy of estimated phylogenetic trees from molecular data. J. Mol. Evol. 19(2): 153-170. 1983.

[20] ORGANIZACIÓN DE LAS NACIONES UNIDAS PARA LA ALIMENTACIÓN Y LAAGRICULTURA (FAO). Plan de Acción Mundial sobre los Recursos Zoogenéticos y la Declaración de Interlaken. Roma: Comisión de Recursos genéticos para la Alimentación y la Agricultura Pp. 19-23. 2007.

[21] ORGANIZACIÓN DE LAS NACIONES UNIDAS PARA LA ALIMENTACIÓN Y LA AGRICULTURA (FAO). Una nueva herramienta en línea para proteger mejor los recursos zoogenéticos. 2017. Roma, Italia. En Línea: https://bit. Iy/2U1V4AW 23-03-2021.
[22] OTA, T. DISPAN: Genetic Distance and Phylogenetic Analysis. Genetic Software. United States of America, Pennsylvania State University, University Park, PA. 6 pp. 1993.

[23] POSADA, S.; SOLARTE, C.; NOGUERA, R. Efecto de la línea genética y el sexo sobre el crecimiento en cuyes (Cavia porcellus). Liv. Res. Rural Develop. 27(1):c1-11. 2015.

[24] RAYMOND, M.; ROUSSET, F. GENEPOP 4.7: Population genetics software for exact test and ecumenicism. J. Hered. 86(3): 248-249. 1995.

[25] ROSALES, C.; CEDILLO, J.; TABOADA, J.; NIETO, P.; QUIZHPI, J.; GUEVARA, G. Comparación de camadas de cobayas (Cavia porcellus) de genotipos ecuatorianos y la línea mejorada Perú. Rev. Prod. Anim. 31(2): 23-26. 2019.

[26] SAITOU, N.; NEI, M. The neighbor-joining method: a new method for reconstructing phylogenetic trees. Mol. Biol. Evol. 4(4): 406-425. 1987.

[27] SOLARTE, C.; ROSERO, C.; BURGOS, W.; ZAMBRANO, G.; ERAZO, Y.; MEJIA, F. El cuy genético. Liv. Res. Rural. Develop. 22(5): párr 21. 2010.

[28] SPONENBERG, D. La Pureza Racial y la Conservación de Recursos Criollos en los Estados Unidos. Act. Iberoam. Cons. Anim. 2: 35-41. 2012.

[29] SPOTORNO, A.; VALLADARES,J.; MARÍN, J.; ZEBALLOS, H. Molecular diversity among domestic guinea-pigs (Cavia porcellus) and their close phylogenetic relationship with the Andean wild species Cavia tschudii. Rev. Chil. Hist. Nat. 77(2): 243-250. 2004.

[30] SWOFFORD, D.; SELANDER, R. BIOSYS-2: A computer program for the analysis of allelic variation in population genetics and biochemical systematics (Release 2.0). University of Illinois, Urbana, Champaign, IL. 1999.

[31] TAMMONE, M. Pérdida de la Diversidad Genética: Implicaciones para la Evolución y la Conservación de dos Especies de Ctenomys (RODENTIA, CTENOMYDAE) en Patagonia Norte. Mastoz. Neotrop. 23(1): 72-73. 2016.

[32] VARGAS, A.; GUTIÉRREZ, R.; MAMANI, M. Una Aplicación del Muestreo de Gibbs en la Estimación de Parámetros Genéticos en Cuyes Utilizando MCMCglmm. Rev. Inv. Vet. Perú. 26(2): 182-188. 2015.

[33] ZAVALA, M. Los Recursos Zoogenéticos, ¿Qué son y Cuál es su Importancia? Saber Más. 30(2): 16-18. 2012. 
